\title{
The entropy production rate a bridge between thermodynamics and chemical kinetics
}

\author{
J.M. Nieto-Villar ${ }^{a, *}$, J. Rieumont $^{a}$ and R. Mansilla ${ }^{b}$ \\ ${ }^{a}$ Departamento de Química-Física, Grupo A. Alzola de Termodinámica de los Sistemas Complejos de La Cátedra Lomonosov, \\ Universidad de La Habana, La Habana 10400 Cuba. Miembro Correspondiente de la Academia Mexicana de Ciencias. \\ e-mail: nieto@fq.uh.cu \\ https://orcid.org/0000-0002-7214-1940 \\ ${ }^{b}$ Centro de Investigaciones Interdisciplinarias en Ciencias y Humanidades, UNAM, México.
}

Received 21 July 2021; accepted 21 August 2021

It is shown how through the entropy production rate a natural unification between the formalism of classical thermodynamics and chemical kinetics is achieved. It is also shown how the entropy production rate represents an alternative way to the sensitivity analysis method in order to determine the fundamental steps in a reaction mechanism.

Keywords: Irreversible thermodynamics; entropy thermodynamics; evolution; entropy in education; science in school.

DOI: https://doi.org/10.31349/RevMexFisE.19.010212

\section{Introduction}

Traditionally, the idea that thermodynamics deals with equilibrium and the chemical kinetics of change has been widely accepted [1]. While to some extent this classification is correct within the framework of classical thermodynamics, where time appears implicitly, it leads undoubtedly to an apparent divorce between thermodynamics and kinetics.

Consequently, in undergraduate courses, students face a dilemma in the study of chemical reactions.

This apparent divorce between thermodynamics and chemical kinetics can be resolved naturally through the thermodynamic formalism of irreversible processes, in particular through the production of entropy per unit time [2].

This communication aims to show how through the entropy production rate, on the one hand, the formalism of classical thermodynamics and chemical kinetics is unified. On the other hand, it can be used in chemical kinetics as an alternative method to that of the sensitivity analysis for the selection of the fundamental steps in a reaction mechanism.

The paper is organized as follows: we first briefly review the basic definitions in classical thermodynamics in Thermodynamics framework section. Section The method of dominating steps, focuses on the development of a thermodynamic outline, based on the rate of entropy production in chemical kinetics as an alternative method to sensitivity analysis for the selection of the fundamental steps in a reaction mechanism. Finally, some comments and remarks are presented in the Conclusion.

\section{Thermodynamics framework}

Unlike other quantities widely used in thermodynamics, such as work, heat, etc., entropy has always been a "dark" and controversial subject within thermodynamics [3-7].
According to De Donder [8] the entropy change of a system $d S_{s}$ is given by

$$
d S_{s}=\delta S_{e}+\delta S_{i}
$$

where $\delta S_{e}$ is entropy flow, that is, the entropy exchanged with the surroundings, while $\delta S_{i}$ is the entropy production due to irreversible processes. Thus, the fundamental postulate of the Second Law establishes that every system evolves in the direction of $\delta S_{i}>0$, and reaches equilibrium when $\delta S_{i}=0$ [8].

For an evolving system subjected to constraints, for example temperature $T$ and the pressure $P$ constants, the entropy production $\delta S_{i}$ can be evaluated through the variation of Gibbs's free energy [8] $d G_{T_{p}}$ as:

$$
\delta S_{i}=-\frac{1}{T} d G_{T_{p}}
$$

The temporal variation of the expression of Eq. (1) represents the entropy production rate as:

$$
\frac{\delta S_{i}}{d t}=-\frac{1}{T} \frac{d G_{T_{p}}}{d t},
$$

where $\delta S_{i} / d t \equiv \dot{S}_{i}$ represents the entropy production rate [2]. The term $d G_{T_{p}} / d t$ can be developed by means of the chain rule as a function of the degree of advance of the reaction $\xi$ as:

$$
\frac{d G_{T_{p}}}{d t}=\left(\frac{\partial G}{\partial \xi}\right)_{T_{P}} \frac{d \xi}{d t},
$$

where $(\partial G / \partial \xi)_{T_{P}}$, according to De Donder and Van Rysselberghe [9] represents the affinity $A \equiv-(\partial G / \partial \xi)_{T_{P}}$, and the term $d \xi / d t$ is the reaction rate $\xi$. The rate of entropy production Eq. (3) can be written as:

$$
\dot{S}_{i}=\frac{1}{T} A \dot{\xi}=-\frac{1}{T} \Delta G \dot{\xi}
$$


where $A=-\Delta G[8]$.

The formula, Eq. (5) establishes a bridge between classical thermodynamics, "thermodynamics force" $\frac{1}{T} A$ and chemical kinetics, the "flow" $\dot{\xi}$ the reaction rate. The affinity $A$ can be evaluated from the isotherm of the reaction [10] by the equation:

$$
A=R T \ln K_{C}-R T \sum_{i=1}^{k} v_{k} \ln C_{k}=R T \ln \left(\frac{K_{C}}{\Pi C_{k}^{v_{k}}}\right),
$$

where $K_{C}=k_{f} / k_{b}$ is the Guldberg-Waage constant, $R$ is the gas constant, $k_{f}, k_{b}$ are the specific rate constants of the forward and backward reaction steps respectively, $C_{k}$ is the concentration of the $k$-th specie, and the $v_{k}$ are the stoichiometric coefficients that are taken, by agreement, as positive for the products and negative for the reactants.

Therefore, the Eq. (6) can be written as:

$$
A=R T \ln \left(\frac{k_{f} \Pi C_{k(f)}^{v_{k}(f)}}{k_{b} \Pi C_{k(b)}^{v_{k}(b)}}\right) .
$$

The rate of reaction $\dot{\xi}$ for an elementary step of reaction can be written as:

$$
\dot{\xi}=\left(\dot{\xi}_{f}-\dot{\xi}_{b}\right)=k_{f} \Pi C_{k(f)}^{v_{k}(f)}-k_{b} \Pi C_{k(b)}^{v_{k}(b)},
$$

where $\dot{\xi}_{f}, \dot{\xi}_{b}$ are the forward and reverse reaction rate respectively. Substituting formulas, Eq. (8) and Eq. (7) in to Eq. (5) is obtained:

$$
\dot{S}_{i}=R\left(\dot{\xi}_{f}-\dot{\xi}_{b}\right) \ln \frac{\dot{\xi}_{f}}{\dot{\xi}_{b}} \geq 0 .
$$

The Eq. (9) analogously to Eq. (5) ends the controversy related to the apparent "divorce" between classic thermodynamics and chemical kinetics.

\section{The method of dominating steps}

It is known that the sensitivity analysis of differential equations has been used successfully in the study of reaction mechanisms in chemical kinetics [11]. Edelson's pioneering works [12-14] they allowed not only the determination of the fundamental reaction steps in a mechanism, but also its reduction. Later, Turanyi $[15,16]$ implemented the method, achieving a drastic reduction in the mechanism of the famous Belousov-Zhabotinsky reaction (BZ), (GTF) model [17] from 80-reaction steps to 42-reation steps.

As an alternative method to the sensitivity analysis, we proposed the use of the entropy production rate based on Eq. (9), a so-called by us Method of the Dominating Steps [18]. For this; we postulate that those reaction steps that exhibit a higher entropy production value would be the fundamental ones in a reaction mechanism.

Let be a mechanism composed of n-reaction steps and $m$ species, represented by (10) such that

$$
\begin{gathered}
x_{1 / i}=x_{2 / i} \\
\vdots \\
x_{m-1 / n}=x_{m / n} .
\end{gathered}
$$

Thus, we have that the entropy production rate of the $\mathrm{n}$ reaction step is given by

$$
\dot{S}_{i / n}=R\left(\dot{\xi}_{f / n}-\dot{\xi}_{b / n}\right) \ln \frac{\dot{\xi}_{f / n}}{\dot{\xi}_{b / n}} \geq 0 .
$$

TABLE I. The entropy production rate of the fundamentals reaction steps for reduced GTF model (adapted from [18]).

\begin{tabular}{cccc}
\hline Reaction step* & $\dot{S}_{i}\left(\frac{\mathrm{J}}{\mathrm{s} \cdot \mathrm{K}}\right)$ & Reaction step* $^{*}\left(\frac{\mathrm{J}}{\mathrm{s} \cdot \mathrm{K}}\right)$ \\
\hline 1 & $4.7 \times 10^{-4}$ & 17 & $1.8 \times 10^{-4}$ \\
2 & $2.9 \times 10^{-4}$ & 21 & $1.2 \times 10^{-6}$ \\
3 & $4.5 \times 10^{-6}$ & 22 & $1.3 \times 10^{-3}$ \\
5 & $2.0 \times 10^{-6}$ & 24 & $5.3 \times 10^{-6}$ \\
7 & $2.0 \times 10^{-6}$ & 26 & $6.3 \times 10^{-6}$ \\
9 & $8.6 \times 10^{-5}$ & 29 & $1.1 \times 10^{-6}$ \\
10 & $7.8 \times 10^{-5}$ & 38 & $1.6 \times 10^{-6}$ \\
11 & $2.6 \times 10^{-3}$ & 39 & $3.7 \times 10^{-6}$ \\
12 & $2.6 \times 10^{-3}$ & 46 & $2.6 \times 10^{-6}$ \\
13 & $3.1 \times 10^{-5}$ & 56 & $2.6 \times 10^{-6}$ \\
15 & $2.1 \times 10^{-5}$ & 61 & $2.4 \times 10^{-6}$ \\
& $7.0 \times 10^{-4}$ & 71 & $1.2 \times 10^{-6}$ \\
\end{tabular}


The $n$-reaction step will be dominant compared to $(n-1)$ reaction step if it is true that: $\dot{S}_{i / n}>\dot{S}_{i / n-1}$. To illustrate the use and efficacy of the Method of the Dominating Steps, as an example, we will show its use in the reduction of the original GTF17 model with 80-reaction steps to 26-reaction steps $[18,19]$ as shown in Table I. An essential condition is that the selected reaction steps reproduce the observed experimental behavior [19].

On the one hand, it should be noted that the 26-reaction steps selected are among the 42-reaction steps set by Turanyi et al. [17] through the use of the sensitivity analysis to the GTF model. On the other hand, this drastic reduction not only incorporates the full richness of the pioneering work of Field et al. [20] (FKN model), periodic oscillations, and it is enough to account, in particular, for the experimental results reported by Ruoff [21], chaotic behavior.

Finally, we have successfully extended the method to the study of the glycolysis mechanism of cancer [22-25]. In the last year, glycolysis of cancer has been a target in oncology [26] Most tumor cells show a higher glycolytic rate than normal cells; this phenomenon is known in the literature as the Warburg's effect. The main objective was to identify reaction steps that can be potential targets in cancer treatment.

From the model proposed by Marín et al. [27] for HeLa and AS-30D tumor cells, 9-reaction steps were identified out of 20-reaction steps. In this sense, 3 of them coincide with the so-called "metabolic control points"; the other six have been identified as potential targets in cancer treatment [28-30].

\section{Conclusions}

In summary, in this paper we arrive at the following conclusions:

1. The rate of entropy production, as a generalization of the Second Law of thermodynamics, represents per se an extreme criterion that marks the directionality of natural processes on a macroscopic scale and constitutes a bridge between the formalism of classical thermodynamics and the chemical kinetics.

2. In biophysical-chemical systems, the rate of entropy production, as a non-extreme criterion, generalizes the so-called "maximum entropy criterion" [31] and constitutes a complementary method to the sensitivity analysis of differential equations, which allows determining the fundamental reaction steps in a reaction mechanism.

\section{Acknowledgments}

Prof. Dr. A. Alzola and R. Quintana in memoriam. To our beloved Alma Mater of the University of Havana. Finally, the authors thank the anonymous reviewers for their helpful comments and interesting suggestions.
1. P. Atkins and J. De Paula, Atkins' Physical Chemistry, (ed.; OUP Oxford, 2014).

2. I. Prigogine, Introduction to thermodynamics of irreversible processes, (ed.; New York: Interscience, 1965).

3. F. L. Lambert, Entropy is simple, qualitatively, Journal of Chemical Education. 79 (2002) 1241

4. F. L. Lambert, The misinterpretation of entropy as disorder, Journal of Chemical Education 89 (2012) 310.

5. F. Flores Camacho, N. Ulloa Lugo, and H. Covarrubias Martínez. "The concept of entropy, from its origins to teachers." Rev. Mex. Fis. E 61 (2015) 69.

6. A. Ben-Naim, Entropy and Information Theory: Uses and Misuses, Entropy 21 (2019) 2112.

7. M. Ribeiro et al., The entropy universo, Entropy 23.2 (2021) 222.

8. D. Kondepudi, I. Prigogine, Modern thermodynamics: from heat engines to dissipative structures, (ed.; John Wiley and Sons, 2014).

9. T. De Donder, P. Van Rysselberghe, Thermodynamic theory of affinity: A book of principles, (ed.; Stanford university press, 1936).

10. R. Mansilla, J.M. Nieto-Villar, La Termodinámica de los sistemas complejos, (ed.; UNAM, 2017).
11. A. Varma, M. Morbidelli, H. Wu, Parametric sensitivity in chemical systems, (ed.; Cambridge University Press; 2005).

12. D. Edelson, V.M. Thomas, Sensitivity analysis of oscillating reactions. 1. The period of the Oregonator, The Journal of Physical Chemistry. 85 (1981) 1555.

13. D. Edelson, D.L. Allara, A computational analysis of the alkane pyrolysis mechanism: Sensitivity analysis of individual reaction steps, International Journal of Chemical Kinetics 12 (1980) 605 .

14. D. Edelson, Sensitivity analysis of proposed mechanisms for the Briggs-Rauscher oscillating reaction, The Journal of Physical Chemistry 87 (1983) 1204.

15. T. Turányi, Sensitivity analysis of complex kinetic systems. Tools and applications, Journal of mathematical chemistry 5 (1990) 203.

16. L. Gyorgy, T. Turányi, and R. J. Field, Mechanistic Details of the Oscillatory Belousov-Zhabotinsky Reaction. J. Phys. Chem. 94 (1990) 7162.

17. T. Turányi, L. Gyorgy, R.J. Field, Analysis and Simplification of the GTF Model of the Belousov-Zhabotinsky Reaction, $J$. Phys. Chem. 97 (1993) 1931.

18. J. Rieumont-Briones, J.M. Nieto-Villar, J.M. García, The Rate of Entropy Production as a Mean to Determine the Most Important Reaction Steps in Belousov-Zhabotinsky Reaction, Anales de Química, International Edition 93 (1997) 147. 
19. J.M. Nieto-Villar, M.G. Velarde, Chaos and Hyperchaos in a Model of the Belousov-Zhabotinsky Reaction in a Batch Reactor, Journal of Non-Equilibrium Thermodynamics 25 (2001) 269.

20. R.J. Field, E. Körös, R. Noyes, Oscillations in Chemical Systems. II. Thorough Analysis of Temporal Oscillation in the Bromate-Cerium-Malonic Acid System, J. Am. Chem. Soc. 94 (1972) 8649,

21. P.J. Ruoff, Chaos in Batch Belousov-Zhabotinsky Systems, $J$. Phys. Chem. 96 (1992) 9104.

22. J.M. Nieto-Villar, E. Izquierdo-Kulich, R. Quintana, and J. Rieumont, Una aproximación del criterio evolutivo de Prigogine a sistemas químicos, Rev. Mex. Fis., 59 (2013) 527.

23. A. Guerra et al., The entropy production in the glycolysis of cancer, Revista Cubana de Física, 31 (2014) 103.

24. S. Montero et al., Cancer glycolysis I: entropy production and sensitivity analysis in stationary state, J. Adenocarcinoma $\mathbf{1}$ (2016) 1-7.

25. S. Montero, R. Martin, R. Mansilla, G. Cocho, J.M. NietoVillar, Parameters Estimation in Phase-Space Landscape Re- construction of Cell Fate: A Systems Biology Approach. In Systems Biology (ed.; Humana Press, New York, NY, 2018; pp 125-170).

26. R.A. Gatenby, R.J. Gillies, Glycolysis in cancer: a potential target for therapy, Int J Biochem Cell Biol 39 (2007) 1358.

27. A. Marín-Hernández et al., Biochimica et Biophysica Acta 1807 (2011) 755, doi:10.1016/j.bbabio.2010.11.006

28. S. Fais, G. Venturi and B. Gatenby, Microenvironmental acidosis in carcinogenesis and metastases : new strategies in prevention and therapy, Cancer (2014) 1095, doi: $10.1007 /$ s10555-014-9531-3

29. H. Pelicano, D.S. Martin, R.H. Xu, P. Huang, Glycolysis inhibition for anticancer treatment, Oncogene 25 (2006) 4633.

30. N. Draoui and O. Feron, Lactate shuttles at a glance: from physiological paradigms to anti-cancer treatments, Disease models and mechanisms 4 (2011) 727, doi: $10.1242 / \mathrm{dmm} .007724$

31. L.M. Martyushev, V.D. Seleznev, Maximum entropy production principle in physics, chemistry and biology, Phys Rep. 264 (2006) 1-45. 